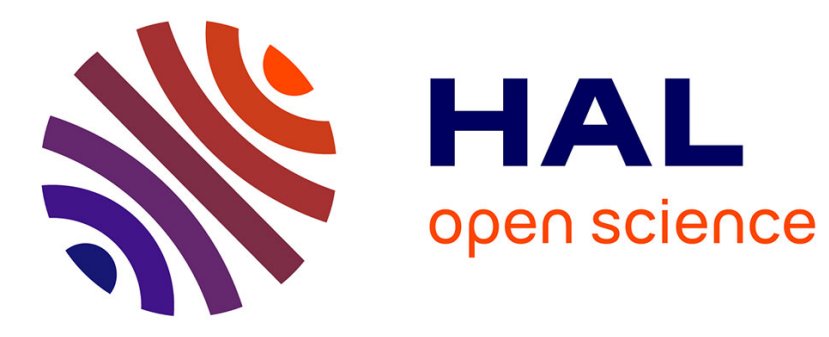

\title{
On the origin of epileptic High Frequency Oscillations observed on clinical electrodes
}

\author{
M. Shamas, P. Benquet, I. Merlet, M. Khalil, W. El Falou, A. Nica, F. \\ Wendling
}

\section{- To cite this version:}

M. Shamas, P. Benquet, I. Merlet, M. Khalil, W. El Falou, et al.. On the origin of epileptic High Frequency Oscillations observed on clinical electrodes. Clinical Neurophysiology, 2018, 129 (4), pp.829841. 10.1016/j.clinph.2018.01.062 . hal-01740202

HAL Id: hal-01740202 https://hal-univ-rennes1.archives-ouvertes.fr/hal-01740202

Submitted on 6 Jul 2018

HAL is a multi-disciplinary open access archive for the deposit and dissemination of scientific research documents, whether they are published or not. The documents may come from teaching and research institutions in France or abroad, or from public or private research centers.
L'archive ouverte pluridisciplinaire $\mathbf{H A L}$, est destinée au dépôt et à la diffusion de documents scientifiques de niveau recherche, publiés ou non, émanant des établissements d'enseignement et de recherche français ou étrangers, des laboratoires publics ou privés. 


\section{On the origin of epileptic High Frequency Oscillations observed on clinical electrodes}

Mohamad SHAMAS ${ }^{1,2,3}$, Pascal BENQUET ${ }^{1,2}$, Isabelle MERLET ${ }^{1,2}$, Mohamad KHALIL $^{3,5}$, Wassim EL FALOU ${ }^{3,5}$, Anca NICA ${ }^{1,4}$, Fabrice WENDLING ${ }^{1,2}$

1 INSERM U1099, LTSI, Campus de Beaulieu - 35042 Rennes Cedex - France

2 Université de Rennes 1, LTSI, Campus de Beaulieu - 35042 Rennes Cedex - France

3 AZM center-EDST, Lebanese University, El mitein street, 1300 Tripoli, Lebanon

4 Neurology Dpt, Functional Explorations Dpt, Epilepsy Surgery Unit, CHU Rennes 16 bd

Bulgarie, 35203 RENNES CEDEX 2, France

5 CRSI research center, Faculty of Engineering, Lebanese University, al-Kubba street, 1300

Tripoli Lebanon

\section{Corresponding Author:}

Fabrice Wendling

INSERM U1099 -LTSI - UNIVERSITE DE RENNES 1

Campus de Beaulieu - 35042 Rennes Cedex - France

Tel:(33) 223235605 or (33) 223236220 - Fax : (33) 223236917

Email: fabrice.wendling@univ-rennes1.fr - http://perso.univ-rennes1.fr/fabrice.wendling/ 


\section{Abstract}

Objective: In this study we aim to identify the key (patho)physiological mechanisms and biophysical factors which impact the observability and spectral features of High Frequency Oscillations (HFOs).

Methods: In order to accurately replicate HFOs we developed virtual-brain / virtual-electrode simulation environment combining novel neurophysiological models of neuronal populations with biophysical models for the source/sensor relationship. Both (patho)physiological mechanisms (synaptic transmission, depolarizing $\mathrm{GABA}_{\mathrm{A}}$ effect, hyperexcitability) and physical factors (geometry of extended cortical sources, size and position of electrodes) were taken into account. Simulated HFOs were compared to real HFOs extracted from intracerebral recordings of 2 patients.

Results: Our results revealed that HFO pathological activity is being generated by feed-forward activation of cortical interneurons that produce fast depolarizing GABAergic post-synaptic potentials (PSPs) onto pyramidal cells. Out of phase patterns of depolarizing GABAergic PSPs explained the shape, entropy and spatiotemporal features of real human HFOs.

Conclusions: The terminology "high-frequency oscillation" (HFO) might be misleading as the fast ripple component $(200-600 \mathrm{~Hz})$ is more likely a "high-frequency activity" (HFA), the origin of which is independent from any oscillatory process.

Significance: New insights regarding the origins and observability of HFOs along depth-EEG electrodes were gained in terms of spatial extent and 3D geometry of neuronal sources.

Keywords: High frequency oscillations, Depolarizing GABA, Depth-EEG, Neuronal population model, Generation mechanisms. 


\section{Highlights}

- High-frequency oscillations (HFOs) on depth-EEG clinical macroelectrodes originate from non-oscillatory post-synaptic processes.

- The synchronization level of neuronal populations explains the switch from HFOs to epileptic spikes.

- Observability of HFOs depends on intracerebral electrode position relative to cortical 3D geometry. 
Abbreviations:

EZ: Epileptogenic Zone

PCs: Pyramidal Cells

FPs: Lead Field Potentials

PSPs: Post-Synaptic Potentials

dGABA: depolarizing GABA

HFA: High Frequency Activity 


\section{Introduction}

In drug-resistant partial epilepsies, resective surgery is the treatment of choice to suppress seizures, provided that the epileptogenic zone (EZ) is clearly identified and that it can be safely removed. In this context, the capacity to rely on objective biomarkers of the EZ is fundamental to define the optimal surgical approach in the specific context of each patient. Prior to surgery, patients may benefit from pre-surgical investigations, among which depthEEG recordings which play a key role as the use of intracranial electrodes offer the unique opportunity to directly and locally record field potentials from brain areas with an excellent temporal resolution (sub-millisecond). Thus depth-EEG signals convey crucial information in the form of electrophysiological markers that must be "decoded" in order to get insight into pathophysiological processes characterizing the EZ.

Among these markers, high-frequency oscillations (HFOs) that occur in the $120-600 \mathrm{~Hz}$ frequency range, on depth-EEG electrodes, have been a topic of increasing interest for the past twenty years (Staba et al. , 2014) since the early clinical reports in mesial temporal lobe epilepsy (Bragin et al., 1999) that were then followed by many clinical studies demonstrating the potentially-high diagnostic value of HFOs in focal epilepsies (Cimbalnik et al. , 2016). However, the relationship between the EZ, on the one hand, and the features of HFOs as observed on multi-contact macroelectrodes classically used for pre-surgical evaluation, on the other hand, is not straightforward and there is still a lack of understanding on the specific information that is carried by HFOs (Engel et al. , 2012). This situation is explained by both neurophysiological and biophysical factors.

From a neurophysiological viewpoint, various neuronal mechanisms have been showed to contribute to both physiological and pathological oscillations in the ripple (120-250 Hz) and in the fast ripple $(250-600 \mathrm{~Hz})$ frequency bands (Jefferys et al. , 2012), including the 
contribution of action potentials during weakly-synchronized firing of pyramidal cells (PCs) (Foffani et al. , 2007) or inhibitory synaptic potentials occurring rhythmically in the perisomatic region of PCs (Shiri et al. , 2015), or both.

From a biophysical viewpoint, many factors are likely to impact the local field potentials (LFPs) recorded at the level of depth electrodes, including the size and geometry of electrode contacts (Worrell et al. , 2008, Crepon et al. , 2010).

So far, very few studies have specifically addressed the issue of the observability of HFOs as recorded with clinical macroelectrodes with no additional embedded microcontacts. Since the pioneer work of Bancaud and Talairach (Bancaud et al. , 1973), these electrodes are classically used for depth-EEG investigation prior to surgery. They differ with other "hybrid electrode" designs like, for instance, the UCLA depth electrode (Bragin et al. , 2002) (Staba et al. , 2002) that includes 4 to $940-\mu \mathrm{m}$ microwires (for multi-unit activity) in addition to millimetric macrocontacts (for LFPs). A previous study conducted by Worrell et al. showed significant differences in terms of frequency and detection rate of fast ripples simultaneously recorded by micro- and macroelectrodes (Worrell et al. , 2008). Therefore, when recorded by macro-electrodes, HFOs can hardly be explained by a major contribution of action potentials, as it is the case when microelectrodes are being used, as reported in many in vivo (Bragin et al. , 1999) or in vitro (Foffani et al. , 2007) studies. Consequently, the origin of macroelectrode-recorded HFOs remains an open question that is addressed in this study.

In order to jointly account for (patho)physiological mechanisms and for biophysical factors, we combined novel computational models of neural masses with virtual brain and virtual electrode models for accurately replicating HFOs as observed on clinical macro-electrodes used in presurgical evaluation of patients with pharmaco-resistant epilepsy.

From this modeling approach, we report new insights regarding the observability of HFOs along clinical depth-EEG macroelectrodes. Key mechanisms involve feed-forward activation 
of interneurons mediating depolarizing $\mathrm{GABA}_{\mathrm{A}}$ post-synaptic potentials and key factors include the spatial extent and the 3D geometry (open- vs. closed field) of neuronal sources comprised in recorded cortical areas.

\section{Materials and Methods}

\subsection{Data Acquisition}

Depth-EEG intracerebral signals were stereotaxically recorded from two patients suffering from drug resistant epilepsy in the Neurology department of the University Hospital of Rennes in France. The monitoring with intracranial electrodes was conducted during the presurgical evaluation of these patients in order to accurately delineate the epileptogenic zone and evaluate the patient's candidacy for surgery. Both patients gave informed approval for participation in research studies. A 256-channel Brain Quick® Micromed System was used to record depth-EEG signals at a sampling rate of $2048 \mathrm{~Hz}$. A hardware high pass filter with 0.1 $\mathrm{Hz}$ as cutoff frequency was used to eliminate the offset of the base line. Intracerebral electrodes were composed of 8 to 18 cylindrical contacts (DIXI Microdeep electrodes, length: $2 \mathrm{~mm}$, diameter: $0.8 \mathrm{~mm}, 1.5 \mathrm{~mm}$ apart, platine-iridium). Electrode insertion points for the first patient are given in Fig. 1A and B. For second patient, information is provided in supplementary Figure SF1. The detection of HFOs was done according to four steps. First the data for all electrodes were examined on an EEG reviewing software developed in our Lab (Amadeus (Wendling, 2015)), and contacts showing clear HFOs on the ongoing background were chosen (Fig. 1C and D). Then the three hours data sets were fed to an automatic detector to detect and classify fast activities into five classes, namely gamma, high gamma, ripples, fast ripples and spike-artifact (Jrad et al. , 2017). Finally, ripples (120-200 Hz) and fast ripples $(200-600 \mathrm{~Hz})$ were visually examined and 50 HFOs from each patient (total of $100 \mathrm{HFOs}$ ) were extracted to be used as a template for target signals (Fig. 1E and F). 


\subsection{HFO computational model: spatial features}

Real post-implantation 3D Magnetic Resonance Imaging (MRI) images for patients who underwent stereotaxic EEG (Bancaud et al. , 1970) were used to construct high resolution triangulated meshes describing with high degree of precision the anatomical structure of the cerebral cortex. To proceed, the open source software "freesurfer" (Fischl, 2012) was used to segment the 3D MRI images, extract gray matter and build a triangulated mesh. This mesh was converted (vtk file format) to be used afterwards in the virtual brain environment specifically designed for simulating depth-EEG signals. The epileptic region responsible for the generation of epileptiform activity was modelled by spatially adjacent elementary triangles each of an average area of $0.5 \mathrm{~mm}^{2}$. Due to this high resolution, we were able to delineate relatively small compact cortical areas corresponding to abnormal brain regions with area ranging from 25 to $60 \mathrm{~mm}^{2}$ i.e. 50 to 120 neural populations. To obtain more realistic HFOs, we considered a larger cortical patch surrounding the epileptic cluster for the background activity. We managed to match virtual electrodes to the exact geometry of the real intracranial electrodes in terms of contact length $(2 \mathrm{~mm})$, number of contacts (10-15), diameter $(0.8 \mathrm{~mm})$, position and orientation relative to the cortical surface.

\subsection{HFO computational model: temporal dynamics}

The electrical contribution of each neural population was represented by a dipole located at the center of each mesh triangle and oriented perpendicularly to its surface. The time varying potential induced by each dipole is determined as the output of an existing neural mass model previously used for simulating various types of signals such as sporadic spikes, rhythmic discharges, normal and ictal activity (Wendling et al. , 2002). In order to generate HFOs several modifications of this previous model were achieved. Firstly, we implemented the feedforward inhibition which is shown to be a crucial element in the fundamental organization of brain circuits entitled for transfer of information between the thalamus and the 
cortex (Womelsdorf et al. , 2014). In line with previous modeling studies (Mina et al. , 2013), the excitatory input activating the PCs also activates the interneurons. Secondly, we considered the postsynaptic downregulation of chloride transfer, known as depolarizing GABA, which has been detected in many neurological disorders, including epilepsy, and was proved to be necessary for generating epileptic discharges in a recently-published computational model (Kurbatova et al. , 2016). Briefly, populations of interneurons receive subcortical excitatory input and generate either feedforward GABAergic excitation or inhibition depending on the (dys)funtional nature of pyramidal cells GABA receptors.

\subsection{Detailed description of the (patho)physiological model}

The model is intended to reproduce the activity recorded by a clinical depth electrode arising from a patch of neocortex (comprising $N$ neuronal populations) in response to subcortical input. All model parameters are documented in Table 1.

2.4.1 Neuronal population model. The neuronal population model structure is detailed in

Fig. 2B. The model consists in 3 types of interacting neuronal sub-populations:

1. Pyramidal Cells (PCs) are the main component of the model from which the LFPs are reconstructed, as the summation of excitatory/inhibitory PSPs. PCs are excited by subcortical input and receive inhibitory PSPs from GABAergic dendritic-projecting $\left(I N_{1}\right)$ and somaticprojecting $\left(\mathrm{IN}_{2}\right)$ local interneurons, as well as abnormal excitatory GABA PSPs in the case where the depolarizing GABA effect is simulated. PCs mediate excitatory PSPs on each and every type of local interneuron and neighboring PCs as well.

2. Dendritic-targeting inhibitory local Interneurons $\left(I N_{1}\right)$ mediate inhibitory PSPs onto PCs. They are characterized by a relatively low firing rate and play an important role in the morphology of the overshoot following an HFO. They are excited by PSPs from the PCs and by excitatory input from subcortical regions. 
3. Somatic-targeting Interneurons $\left(I N_{2}\right)$ integrate PSPs from three different sources: glutamatergic EPSPs from PCs, GABAergic IPSPs from $I N_{1}$ and EPSPs mediated by the thalamic input. In healthy tissue, most of somatic-targeting GABAergic interneurons induce IPSPs on PCs. However, it has been shown that in epileptic brain tissue the reversal potential for chloride is shifted toward a more depolarized potential (Barmashenko et al. , 2011), in this case GABAergic PSPs become depolarizing. In the proposed neural mass model, the reversal potential for chloride was not explicitly implemented, rather excitatory GABAergic PSPs onto PCs were represented. In this study, we symbolized this mechanism in subpopulations of interneurons denoted as $I N_{2}^{\prime}$. This hypothesis has already been considered in some computational models of epileptiform activity (see recent review in (Wendling et al. , 2016)). As a result, we considered that PCs were receiving inhibitory GABAergic PSPs from one type of somatic-targeting interneurons denoted by $I_{2}$ and excitatory GABAergic PSPs from a second type denoted by $I N_{2}^{\prime}$. The delineated "epileptic" patch could contain "normal" populations where the somatic-targeting interneurons are of only $I N_{2}$ type, and "abnormal" populations where the somatic-targeting interneurons are of both $I N_{2}$ and $I N_{2}^{\prime}$ type, thus mediating a dual excitatory/inhibitory GABAergic PSPs on the PCs. Readers may refer to Supplementary Material (SM1) for detailed mathematical description of the model.

2.4.2 Subcortical input. The model input denoted by $\mathrm{P}(\mathrm{t})$ is illustrated in Fig. 2D. It represents the afferent input to the cortical patch modeled as set of signals $\mathrm{P}_{\mathrm{k}}(\mathrm{t})$, each denoting a density of action potentials (APs) targeting a neuronal population in the patch. Each $\mathrm{P}_{\mathrm{k}}(\mathrm{t})$ is a combination of 2 components one of which is a Gaussian noise $N\left(\mu, \sigma_{n}\right)$ with $\mu$ as mean and $\sigma_{n}$ as standard deviation ( $n$ stands for noise), and the other is an short-duration pulse $\Delta \overline{A P}$ that accounts for an abrupt increase-decrease of action potentials (APs). The 
occurrence time of each $\Delta \overline{A P}$ in $\mathrm{P}(\mathrm{t})$ obeys a Gaussian distribution with mean $t_{0}$ (center of simulated HFO) and standard deviation $\sigma_{j}$ (where $j$ stands for "jitter"). This jitter parameter $\sigma_{j}$ allows for controlling the synchronization level of the input.

For a detailed description on the combination of spatial and temporal parameters readers can refer to Supplementary Material section (SM2).

All Signal processing and feature extraction methods are detailed in Supplementary Material (SM3).

\section{Results}

\subsection{Real human HFOs.}

Event of interest were automatically detected using a signal processing method based on Gabor function applied to monopolar depth-EEG signals recorded from depth electrodes (Fig. 1A-C). HFOs were visually validated. Typical examples are shown in Fig. 1D-F. As depicted, HFOs presented as transient events of an average duration of $100 \mathrm{~ms}$ and characterized by a fast oscillation occurring during the decaying phase of a slower large amplitude wave.

\subsection{Simulated HFOs.}

Simulation results are displayed in Fig. 2 (see also the methods section for technical details about the computational model). A patch was defined on the 3D high-resolution mesh of the neocortex. A virtual intracerebral electrode of realistic geometry was inserted in this cortical patch, orthogonally to its surface (Fig. 2A). The local field potential (LFP) of each neuronal population over this patch was simulated by a neural mass model comprising sub-populations of glutamatergic pyramidal cells (PCs) targeted by two types of GABAergic interneurons (INs) producing post-synaptic potentials (PSPs) with slow (IN1, dendritic-projecting) and fast kinetics (IN2, somatic-projecting). In the second sub-population IN2, a subset of interneurons 
(IN2') could be defined to generate excitatory PSPs onto PCs, referred to as depolarizing GABA (dGABA) PSPs. It is worth mentioning that this dGABA effects is a characteristic property of the epileptogenic tissue (see (Kaila et al. , 2014) for review). Based on the dipole theory, the forward problem of depth-EEG was solved (see methods) to simulate signals along the virtual electrode sensors (Fig. 2C). Then, by adjusting the jitter of the densities of excitatory action potentials (Fig. 2D) afferent to the cortical patch, simulated HFOs could be produced (Fig. 2E), showing both the slow and the ripple components (Fig. 2E) that qualitatively resembled actual HFO in shape, duration, and frequency. The kinetics of the PSPs mediated by each of the subpopulations and their overall contribution to the collected PSP on the PCs are illustrated in Supplementary Figure SF2. Simulated responses are consistent with experimentally-recorded values (Banks et al. , 2000) (Wu et al. , 2004) (Struber et al. , 2015).

\subsection{Real vs. simulated HFOs.}

Quantitative comparison is provided in Fig. 3. Both time-series and spectrograms of simulated signals (Fig. 3A) indicated that the model was able to accurately reproduce the features and the diversity of HFOs recorded in patients. This visual inspection of the time-frequency content of analyzed signals was also confirmed by signal processing. The first quantitative index revealed that the ratio of the energy in the fast ripple band $(200-600 \mathrm{~Hz})$ to the total energy (Ehf/Etot) was similar $(p=0.20$, Wilcoxon-test $)$ in both the actual $(n=100)$ and simulated ( $n=360)$ events (Fig. 3B). The second quantitative index (NSE) showed that the irregularity, complexity and randomness in the power spectrum of simulated signals strongly matched the same features in real signals, as no statistical difference ( $\mathrm{p}=0.41$, Wilcoxon-test) was found between NSE values (Fig. 3C). Finally, the third index quantifying the phaseamplitude coupling was found to be similar in simulated and real signals ( $\mathrm{p}=0.49$, Wilcoxontest). This result indicates that the increase of energy associated with the fast ripple 
component occurred at the right phase on the slow component in simulations, when compared to real data.

\subsection{Mechanistic insights on HFO features.}

We performed extensive simulations ( $n=6600$ simulated events) in order to determine what key (patho)physiological mechanisms (represented in the model) were directly impacting the HFO features, namely the energy ratio (Ehf/Etot), the spectral entropy (NSE), the duration of the slow component $(\sigma)$ and the median frequency of the fast ripple component (fmed). As reported in Fig. 4A, the increase of amplitude of PSPs mediated by depolarizing GABA (dGABA) onto pyramidal cells dramatically i) increased both the energy and the median frequency associated with the fast ripple component (Fig. 4-A1 and A2), ii) increased the spectral entropy of simulated HFOs (Fig. 4-A3) and iii) decreased the duration of the slow component (Fig. 4-A4). We then investigated the influence of the percentage of neuronal populations that implement this dGABA effect (Fig. 4B). We found that a minimum percentage of $30 \%$ is a necessary condition to get events with realistic slow and fast components. In addition, and in contrast with Fig. 4A, we found that this percentage had a light impact on the energy ratio (Fig. 4-B1) and on the spectral entropy (Fig. 4-B3) and no effect on the median frequency of the fast ripple component (Fig. 4-B2) and on $\sigma$ (Fig. 4-B4). Finally, the jitter of the excitatory input (Fig. 4C) was also found to have a strong impact on spectral and morphological features (energy ratio and the duration of the slow component, Fig. 4-C1 and C4). No effect was evidenced regarding the spectral entropy and the median frequency (Fig. 4-C2 and C3). Interestingly, as shown in Fig. 4D, when this jitter parameter was gradually reduced from $16 \mathrm{~ms}$ to $3 \mathrm{~ms}$ (while keeping all other parameters constant), a switch from HFO events to epileptic spikes was observed in simulated LFPs. 


\subsection{From multichannel HFOs to source geometry.}

In order to address the central clinical question of why and how the folded nature of the neocortex impacts the observability of HFOs on intracerebral recordings, we performed specific simulations where we could accurately control the electrode position w.r.t. the 3D geometry of the cortical surface. As illustrated in Fig. 5A, there was a strong difference between simulated LFPs obtained in an "open-field" vs. those arising from a "closed-field". In the open-field configuration, dipole vectors are, on average, oriented parallel to each other in such a way that they tend to sum up. In contrast, the closed-field configuration corresponds to the case where these vectors are, to a large extent, oriented opposite to each other and tend to cancel out. In the first case, simulated HFOs were clearly visible on 5 to 6 electrode contacts (Fig. 5A) while they were focalized on 1 to 2 contacts in the closed-field case (Fig. 5B). Interestingly, actual depth-EEG recordings exhibit signal features such as 1) presence/absence of HFOs on contiguous contacts and 2) HFO-to-background ratio, that strongly match simulations, when electrode contacts are in an "open-field" (Fig. 5C) or "closed-field" configuration (Fig. 5D). Finally, we quantified the "HFO-to-BKG" ratio (see methods) in both conditions. As depicted in Fig. 5E, this ratio was higher by $+9 \mathrm{~dB}$, i.e. more than 7 times, when the epileptic source was located over a gyrus than when it was inside a sulcus. As discussed in the next section, the model provided a clear explanation about actual values of the HFO-to-BKG ratio (Fig. 5F) in term of respective contribution of "pathological" sources, producing HFOs, and "normal" sources, producing background activity, onto electrode contacts.

\section{Discussion}

Over the past decade, HFOs (HFOs, $80-600 \mathrm{~Hz}$ ) have been a topic of increasing interest. Regarding the upper frequency band $(250-600 \mathrm{~Hz}$ ), a number of experimental studies, 
combined with computational modeling, have led to a number of hypotheses regarding the cellular and network mechanisms involved in the generation of FRs: i) major contribution of interneurons and non-synaptic gap junction couplings between excitatory cells (Roopun et al. , 2010); ii) “out-of-phase” firing patterns of small clusters of neurons (Foffani et al. , 2007) (Ibarz et al. , 2010) and iii) weakly-synchronized "hyperexcitable" neurons featuring depolarizing GABA IPSPs (Demont-Guignard et al. , 2012). In all these studies, signals were observed at the level of microelectrodes implanted in non-folded brain tissue. In contrast, and despite the increasing number of clinical studies investigating HFOs in patients candidate to surgery, the neurophysiological and biophysical mechanisms governing their observability on clinical depth macroelectrodes has received much less attention.

Progress in this field would lead to better interpretation of HFOs and potential use of the information derived from electrophysiological signals for the care of epileptic patients, typically for the definition of the optimal surgical approach.

In this study, we used computational modeling and simulation of brain signals as an efficient approach to address this issue and get insights about mechanisms of generation of pathological HFOs, on the one side, and observability conditions on clinical electrodes, on the other side.

To the best of our knowledge, the present study is the first aimed at accurately replicating HFOs as recorded by clinical electrodes with physiologically-grounded models. To proceed, we developed a novel computational model at mesoscopic scale. This model fully describes the activity of a neocortical patch recorded by a nearby intracerebral multiple-lead electrode of realistic dimension. It allows for simulation of depth-EEG signals while accounting for both the spatial features and the temporal dynamics of neuronal populations comprised in the considered neocortical patch with folded geometry accurately described by a 3D mesh obtained from MRI data. Interestingly, at the level of cortical neuronal populations, some 
mechanisms potentially impacting HFO features were implemented. Key model features include afferent input with tunable synchronization, feed-forward inhibition, tunable excitability level (via collateral excitation among PCs and slow/fast feedback inhibition) and depolarizing GABA currents. The main findings obtained with this model are summarized in Fig. 6 and discussed below.

\subsection{Post-synaptic origin of macroelectrode-recorded HFOs}

The contribution of APs to pathological HFOs (fast ripples) is an accepted fact. Several studies reported that APs of quasi-synchronously bursting PCs are essential components of HFOs detected on microwires (Bragin et al. , 1999) (Ibarz et al. ,2010) (Dzhala et al. , 2004). The relation between HFOs and APs in LFPs has been investigated in microelectrodes recordings only. In contrast, when macroelectrodes are being used the relationship between membrane potentials at cellular level and depth-EEG signals is more elusive. In this study, we propose a hypothesis alternative to 'APs' for mechanisms leading to HFOs observed on macro-contacts. Due to their large size, both close and far neuronal sources, extending over a considerably large spatial area, contribute to the measured signal. It is admitted that (1) the electric fields produced by APs can only be detected at a proximity of a few tens of micrometers from its source (Bedard et al. , 2006) and that (2) the amplitude of spike-related currents are subjected to a steep decay exponentially proportional to the distance $\left(1 / \mathrm{r}^{\mathrm{n}}\right)$ where $\mathrm{n}>2$ (Pettersen et al. , 2008). In addition, (3) the non-homogenous low-conductive and capacitive nature of the extracellular medium results in a low pass filtering effect. According to the three above considerations, it is unlikely that APs directly contribute to the EEG signal recorded by macro-contacts. In contrast, it is more likely that slower post-synaptic currents recorded farther from the electrode contact significantly mask the attenuated fast transmembrane ionic currents involved in APs (Destexhe et al. , 2013). This hypothesis contrasts with a previous simulation study (Reimann et al. ,2013) which reports that active 
membrane conductance contribute to extracellular LFPs and can surpass the effect of PSPs even for high frequencies beyond $100 \mathrm{~Hz}$. However, it is worth noting that LFPs were estimated on virtual "point" electrodes situated in the extracellular medium almost at the contact of simulated neurons. This contrasts with our model in which (1) the real size of macro-contacts and (2) distant sources are accounted for. Finally, additional evidence is brought by simultaneous microwire and clinical macroelectrode recordings (Worrell et al. , 2008) reporting that HFOs detected by microelectrodes are much faster (very high frequency 350-700 Hz) and more abundant on microelectrodes. Overall, our modeling results, in line with the above studies, suggest that the mechanisms which underlie HFOs observed on microcontacts may differ from those underlying HFOs on macro-contacts.

\subsection{Key role of feed-forward activation of GABAergic interneurons.}

In case of epileptic brain tissue, it is well established that reversal potential for chloride can be depolarized in some pyramidal cells, leading to excitatory synaptic GABAergic PSP (Cohen et al. , 2002) (Huberfeld et al. , 2007) (Ben-Ari et al. , 2012). Depolarizing GABAergic (dGABA) synaptic potential contribute to epileptic spikes (Huberfeld et al. , 2007), highfrequency oscillations (200-600 Hz) (Alfonsa et al. , 2015) and seizure onset (Lillis et al. , 2012).

In previous studies, we already proposed computational models implementing the $\mathrm{dGABA}_{\mathrm{A}}$ effect and showed its contribution to epileptic spikes (Demont-Guignard et al. , 2009), fast ripple (Demont-Guignard et al. , 2012), fast-onset activity (Kurbatova et al. , 2016) and seizure-like activity (Wendling et al. , 2012) (Wendling et al. , 2016).

In the present study, we go beyond and propose a neural mass model implementing both $\mathrm{dGABA}_{\mathrm{A}}$ and feed-forward inhibition as represented by an excitatory input onto somaticprojecting GABAergic interneurons. In addition, the implementation of dGABA followed recently-published optogenetic studies showing that i) selective activation of somatic 
targeting parvalbumin-expressing $(\mathrm{PV}+)$ interneurons generated excitatory GABAergic responses in pyramidal neurons (Ellender et al. , 2014) and ii) activation of basket-cells caused a positive shift in the GABAergic reversal potential measured in pyramidal cells up to $25 \mathrm{mV}$, inducing interictal and ictal epileptic activity (Alfonsa et al. , 2015) (Ellender et al. 2014). The kinetics of the GABAergic fast inhibitory potentials in physiological condition is approximately $1 \mathrm{~ms}$ in rise time and $11 \mathrm{~ms}$ in decay time (Struber et al. , 2015). In fact, as the reversal potential value of chloride directly depends on the ratio between extracellular and intracellular chloride concentration (Nernst equation), but not on channel properties (as for example, the subunit composition of the receptors). Therefore, we kept the same kinetics for depolarizing and hyperpolarizing $\mathrm{GABA}_{\mathrm{A}}$. To the best of our knowledge, such mechanisms have not been jointly considered in computational models of epileptic brain activity.

Very interestingly, this mechanism of feed-forward excitation of somatic-projecting GABAergic interneurons mediating abnormal depolarizing PSPs was found to play a key role in the generation of ultra-fast activity in the fast ripple frequency band (around $350 \mathrm{~Hz}$ ). As shown in Figure 4-A2, tuning the $\mathrm{GABA}_{\mathrm{A}}$ PSPs amplitude linearly affect the dominant frequency of simulated HFOs suggesting that there might exist a "continuum" in the frequency domain instead of distinct frequency bands, as commonly used in the literature.

This result constitutes a sharp prediction strengthened by the accurate replication of morphological and spectral features of actual epileptic HFOs.

\subsection{High-frequency “oscillation" vs. "activity".}

Our modeling results strongly suggest that the high-frequency component in analyzed epileptic events originates from depolarizing fast PSPs onto PCs. These "abnormal" PSPs are generated, in a de-synchronized manner, by some of the neuronal populations distributed over the cortical patch. They directly contribute to the LFP fast component "captured" by clinical macro-electrodes. In this regard, the terminology "high-frequency oscillation" (HFO) is 
somehow misleading as the model indicates that the fast ripple component, regardless of the background activity which may - or may not - be oscillatory, more likely corresponds to a "high-frequency activity" (HFA) that is not related to any underlying oscillatory process.

Similar observations were previously described in studies involving gamma patterns, as reported by Einevoll et al. (Einevoll et al. , 2013) who suggested that high-gamma LFPs may reflect spiking activity rather than genuine network oscillations. This finding is also supported by normalized spectral entropy values computed on our simulated signals that are congruent with normalized values computed on real HFOs and reported elsewhere (Canolty et al. , 2006) (Ibarz et al. ,2010) (Menendez de la Prida et al. , 2011). As compared to a pure oscillation, our results strongly suggest that the observed increased entropy originates from the random nature of HFA, itself being directly related to sharp fast depolarizing PSPs in response to a jitter input (see also next paragraph). In addition, the fact that connectivity among neuronal populations was not a necessary condition in the model to produce HFA was an unexpected result. However, this result is in line with the recent study reported by Gliske et al. (Gliske et al. , 2017) who showed, in a detailed model (cellular level) that narrowband high-frequency oscillations can emerge from completely asynchronous and independent activity generated by neurons contributing to LFPs. Finally, and for the first time, our results explain the different nature of HFA i) as observed on clinical macro-electrodes and stemming from ultrafast desynchronized PSPs "captured" by large (mm scale) sensors in a relatively far field and ii) as observed on experimental micro-electrodes and originating from de-synchronized signals (action potentials and PSPs) "captured" by small ( $\mu \mathrm{m}$ scale) sensors located in the close vicinity of PCs.

\subsection{Afferent input shapes recorded events.}

The random occurrence times of volleys of afferent action potentials (APs) onto neuronal subpopulations, characterized by the jitter parameter, was found to dramatically impact both the 
morphology and the spectral content of simulated HFOs. Specifically, when the jitter increases, the ripple component becomes more prominent and the "bell-shape" slow component widens. In contrast, when the jitter decreases, the morphology of the simulated epileptic event switches from an HFO to a spike. Interestingly, a number of studies have reported that both HFOs and epileptic spikes which are very often observed in patients during intra-cranial recordings (Zelmann et al. , 2009) can occur either in a separate or in a superimposed manner. Our results not only confirm this observation but also go beyond as they provide an explanation for the co-occurrence of these two markers of epilepsy: the temporal characteristics (distribution of AP volleys) of the input afferent to the cortical epileptic zone determines the nature of epileptic events (HFOs vs. epileptic spikes) observed in LFPs.

The synchronization of the afferent input (i.e. the input jitter) may be affected by several factors. First, depending on the power of the input noise, the resting membrane potential of the neurons may increase and get closer to threshold value, leading to increase of firing probability and subsequently increased level of synchronization (Diba et al. , 2004) (Kole et al. , 2006). Second, the vigilance state can also impact the distribution of afferent APs. Typically, during slow-wave sleep (SWS), the cortical input from thalamo-cortcial (TC) cells is modified compared to the awake state, consisting of alternating phases of depolarized UP and hyperpolarized DOWN state (Steriade et al. , 2003). This results in a change of the distribution, in time, of volleys of APs to cortical regions, and possibly to increased jitter value. Interestingly, some studies have reported higher occurrence rates of HFOs during SWS (Frauscher et al. , 2015) (von Ellenrieder et al. , 2016) (Song et al. , 2017). Along the same idea, and in accordance with the "low jitter value" prediction of the model, many studies have reported that epileptic spikes involve the co-activation of distant structures (Bourien et al. , 
2005) with hypersynchronous input from one region to another as, for instance, interictal events recorded from CA1 and driven by CA3 (Avoli, 2014).

\subsection{Brain folded surface impacts HFO observability.}

Our model provided the unique opportunity to explore the intricate relationship between the source geometry and the morphological content of signals collected on contiguous clinical sensors. Results shed light on one essential factor. Indeed, the intuitive view according to which dipolar sources cancel each other in the case of closed-fields is challenged by this study. Our modeling results strongly suggest that the observability of HFOs on clinical sensors is most affected by the distance between sources and sensors. Simulations showed that this distance can be very short between "normal" sources and near-by sensors in the case where the electrode goes through a sulcus (close-field). This configuration results in the masking of HFOs by background activity, as quantified by HFO-to-BKG ratio and as supported by the high similarity of simulated activity along the virtual and actual electrode. This result is of high clinical interest as the model bridges between the content of depth-EEG signals and the electrode trajectory within the 3D complex geometry of targeted brain regions. In addition, the model enhances the interpretation of collected signals showing that "lessvisible" HFOs should not be automatically associated with "less-pathological" sources. 


\subsection{Perspectives}

In its current version the model does not explicitly represent the activity of subcortical regions (such as amygdala, hippocampus, and enthorinal cortex) although these are known to play a role in generating epileptic activities. Technically, the pipeline presented in this study to simulate intracerebral EEG signals can be enhanced to account for deep sources provided that the mesial structures are successfully segmented from 3D MRI images and that the information about spatial features of their cellular configurations is known.

Virtual electrodes were modeled to match real clinical electrodes in shape and size (conducting cylinders separated by insulators) so that the patient-specific SEEG implantation can be reproduced. A simple approach based on infinite volume conductor characterized by uniform conductivity was used to reconstruct SEEG signals recorded at each electrode contact. Therefore, our model does not account for biophysical effects related to the large high conductivity surface in contact with the brain tissue. To overcome this limitation, enhanced models could be considered to account for very close populations to the electrode contacts (von Ellenrieder et al. , 2012) or for the resistive and capacitive effect induced by the electrode-tissue interface (Kent et al. , 2014).

\section{Acknowledgements}

This work received support from the French ANR and GDOS (Project "Vibrations", Programme de Recherche Translationelle en Santé, ANR-13-PRTS-0011) and from the AZM and SAADE Association (Tripoli, Lebanon).

\section{Disclosure}

None of the authors has any conflict of interest to disclose. 


\section{References}

Alfonsa H, Merricks EM, Codadu NK, Cunningham MO, Deisseroth K, Racca C, et al. The contribution of raised intraneuronal chloride to epileptic network activity. J Neurosci. 2015;35:7715-26.

Avoli M. Mechanisms of epileptiform synchronization in cortical neuronal networks. Curr Med Chem. 2014;21:653-62.

Bancaud J, Angelergues R, Bernouilli C, Bonis A, Bordas-Ferrer M, Bresson M, et al. Functional stereotaxic exploration (SEEG) of epilepsy. Electroencephalogr Clin Neurophysiol. 1970;28:85-6.

Bancaud J, Talairach J. [Methodology of stereo EEG exploration and surgical intervention in epilepsy]. Rev Otoneuroophtalmol. 1973;45:315-28.

Banks MI, White JA, Pearce RA. Interactions between distinct GABA(A) circuits in hippocampus. Neuron. 2000;25:449-57.

Barmashenko G, Hefft S, Aertsen A, Kirschstein T, Köhling R. Positive shifts of the GABAA receptor reversal potential due to altered chloride homeostasis is widespread after status epilepticus. Epilepsia. 2011;52:1570-8.

Bedard C, Kroger H, Destexhe A. Model of low-pass filtering of local field potentials in brain tissue. Phys Rev E Stat Nonlin Soft Matter Phys. 2006;73:051911.

Ben-Ari Y, Khalilov I, Kahle KT, Cherubini E. The GABA excitatory/inhibitory shift in brain maturation and neurological disorders. Neuroscientist. 2012;18:467-86.

Bourien J, Bartolomei F, Bellanger JJ, Gavaret M, Chauvel P, Wendling F. A method to identify reproducible subsets of co-activated structures during interictal spikes. Application to intracerebral EEG in temporal lobe epilepsy. Clin Neurophysiol. 2005;116:443-55.

Bragin A, Engel J, Jr., Wilson CL, Fried I, Mathern GW. Hippocampal and entorhinal cortex high-frequency oscillations $(100--500 \mathrm{~Hz})$ in human epileptic brain and in kainic acid--treated rats with chronic seizures. Epilepsia. 1999;40:127-37.

Bragin A, Wilson CL, Staba RJ, Reddick M, Fried I, Engel J, Jr. Interictal high-frequency oscillations $(80-500 \mathrm{~Hz})$ in the human epileptic brain: entorhinal cortex. Ann Neurol. 2002;52:407-15.

Canolty RT, Edwards E, Dalal SS, Soltani M, Nagarajan SS, Kirsch HE, et al. High gamma power is phase-locked to theta oscillations in human neocortex. Science. 2006;313:1626-8.

Cimbalnik J, Kucewicz MT, Worrell G. Interictal high-frequency oscillations in focal human epilepsy. Curr Opin Neurol. 2016;29:175-81.

Cohen I, Navarro V, Clemenceau S, Baulac M, Miles R. On the origin of interictal activity in human temporal lobe epilepsy in vitro. Science. 2002;298:1418-21.

Crepon B, Navarro V, Hasboun D, Clemenceau S, Martinerie J, Baulac M, et al. Mapping interictal oscillations greater than $200 \mathrm{~Hz}$ recorded with intracranial macroelectrodes in human epilepsy. Brain. 2010;133:33-45.

Demont-Guignard S, Benquet P, Gerber U, Biraben A, Martin B, Wendling F. Distinct hyperexcitability mechanisms underlie fast ripples and epileptic spikes. Ann Neurol. 2012;71:342-52.

Demont-Guignard S, Benquet P, Gerber U, Wendling F. Analysis of intracerebral EEG recordings of epileptic spikes: insights from a neural network model. IEEE Trans Biomed Eng. 2009;56:2782-95.

Destexhe A, Bedard C. Local field potential. Scholarpedia. 2013;8:10713.

Diba K, Lester HA, Koch C. Intrinsic noise in cultured hippocampal neurons: experiment and modeling. J Neurosci. 2004;24:9723-33. 
Dzhala VI, Staley KJ. Mechanisms of fast ripples in the hippocampus. J Neurosci. 2004;24:8896-906.

Einevoll GT, Kayser C, Logothetis NK, Panzeri S. Modelling and analysis of local field potentials for studying the function of cortical circuits. Nat Rev Neurosci. 2013;14:770-85.

Ellender TJ, Raimondo JV, Irkle A, Lamsa KP, Akerman CJ. Excitatory effects of parvalbumin-expressing interneurons maintain hippocampal epileptiform activity via synchronous afterdischarges. J Neurosci. 2014;34:15208-22.

Engel J, Jr., da Silva FL. High-frequency oscillations - where we are and where we need to go. Prog Neurobiol. 2012;98:316-8.

Fischl B. FreeSurfer. Neuroimage. 2012;62:774-81.

Foffani G, Uzcategui YG, Gal B, Menendez de la Prida L. Reduced spike-timing reliability correlates with the emergence of fast ripples in the rat epileptic hippocampus. Neuron. 2007;55:930-41.

Frauscher B, von Ellenrieder N, Ferrari-Marinho T, Avoli M, Dubeau F, Gotman J. Facilitation of epileptic activity during sleep is mediated by high amplitude slow waves. Brain. 2015;138:1629-41.

Gliske SV, Stacey WC, Lim E, Holman KA, Fink CG. Emergence of Narrowband High Frequency Oscillations from Asynchronous, Uncoupled Neural Firing. Int J Neural Syst. 2017;27:1650049.

Huberfeld G, Wittner L, Clemenceau S, Baulac M, Kaila K, Miles R, et al. Perturbed chloride homeostasis and GABAergic signaling in human temporal lobe epilepsy. J Neurosci. 2007;27:9866-73.

Ibarz JM, Foffani G, Cid E, Inostroza M, Menendez de la Prida L. Emergent dynamics of fast ripples in the epileptic hippocampus. J Neurosci. 2010;30:16249-61.

Jefferys JG, Menendez de la Prida L, Wendling F, Bragin A, Avoli M, Timofeev I, et al. Mechanisms of physiological and epileptic HFO generation. Prog Neurobiol. 2012;98:250-64. Jrad N, Kachenoura A, Merlet I, Bartolomei F, Nica A, Biraben A, et al. Automatic Detection and Classification of High-Frequency Oscillations in Depth-EEG Signals. IEEE Trans Biomed Eng. 2017;64:2230-40.

Kaila K, Ruusuvuori E, Seja P, Voipio J, Puskarjov M. GABA actions and ionic plasticity in epilepsy. Curr Opin Neurobiol. 2014;26:34-41.

Kent AR, Grill WM. Analysis of deep brain stimulation electrode characteristics for neural recording. J Neural Eng. 2014;11:046010.

Kole MH, Hallermann S, Stuart GJ. Single Ih channels in pyramidal neuron dendrites: properties, distribution, and impact on action potential output. J Neurosci. 2006;26:1677-87.

Kurbatova P, Wendling F, Kaminska A, Rosati A, Nabbout R, Guerrini R, et al. Dynamic changes of depolarizing GABA in a computational model of epileptogenic brain: Insight for Dravet syndrome. Exp Neurol. 2016;283:57-72.

Lillis KP, Kramer MA, Mertz J, Staley KJ, White JA. Pyramidal cells accumulate chloride at seizure onset. Neurobiol Dis. 2012;47:358-66.

Menendez de la Prida L, Trevelyan AJ. Cellular mechanisms of high frequency oscillations in epilepsy: on the diverse sources of pathological activities. Epilepsy Res. 2011;97:308-17.

Mina F, Benquet P, Pasnicu A, Biraben A, Wendling F. Modulation of epileptic activity by deep brain stimulation: a model-based study of frequency-dependent effects. Front Comput Neurosci. 2013;7:94.

Pettersen KH, Einevoll GT. Amplitude variability and extracellular low-pass filtering of neuronal spikes. Biophys J. 2008;94:784-802. 
Reimann MW, Anastassiou CA, Perin R, Hill SL, Markram H, Koch C. A biophysically detailed model of neocortical local field potentials predicts the critical role of active membrane currents. Neuron. 2013;79:375-90.

Roopun AK, Simonotto JD, Pierce ML, Jenkins A, Nicholson C, Schofield IS, et al. A nonsynaptic mechanism underlying interictal discharges in human epileptic neocortex. Proc Natl Acad Sci U S A. 2010;107:338-43.

Shiri Z, Manseau F, Levesque M, Williams S, Avoli M. Interneuron activity leads to initiation of low-voltage fast-onset seizures. Ann Neurol. 2015;77:541-6.

Song I, Orosz I, Chervoneva I, Waldman ZJ, Fried I, Wu C, et al. Bimodal coupling of ripples and slower oscillations during sleep in patients with focal epilepsy. Epilepsia. 2017;58:197284 .

Staba RJ, Stead M, Worrell GA. Electrophysiological biomarkers of epilepsy. Neurotherapeutics. 2014;11:334-46.

Staba RJ, Wilson CL, Bragin A, Fried I, Engel J, Jr. Quantitative analysis of high-frequency oscillations $(80-500 \mathrm{~Hz})$ recorded in human epileptic hippocampus and entorhinal cortex. $\mathbf{J}$ Neurophysiol. 2002;88:1743-52.

Steriade M, Timofeev I. Neuronal plasticity in thalamocortical networks during sleep and waking oscillations. Neuron. 2003;37:563-76.

Struber M, Jonas P, Bartos M. Strength and duration of perisomatic GABAergic inhibition depend on distance between synaptically connected cells. Proc Natl Acad Sci U S A. 2015;112:1220-5.

von Ellenrieder N, Andrade-Valenca LP, Dubeau F, Gotman J. Automatic detection of fast oscillations (40-200 Hz) in scalp EEG recordings. Clin Neurophysiol. 2012;123:670-80.

von Ellenrieder N, Frauscher B, Dubeau F, Gotman J. Interaction with slow waves during sleep improves discrimination of physiologic and pathologic high-frequency oscillations (80$500 \mathrm{~Hz}$ ). Epilepsia. 2016;57:869-78.

Wendling F. Software AMADEUS-Visualisation Version 3.0. IDDN: FR.001.420017.000.S.P.2015.000.31230. Paris: Agence Pour la Protection des Programmes, Inserm - Univ Rennes; 2015.

Wendling F, Bartolomei F, Bellanger JJ, Chauvel P. Epileptic fast activity can be explained by a model of impaired GABAergic dendritic inhibition. Eur J Neurosci. 2002;15:1499-508.

Wendling F, Bartolomei F, Mina F, Huneau C, Benquet P. Interictal spikes, fast ripples and seizures in partial epilepsies--combining multi-level computational models with experimental data. Eur J Neurosci. 2012;36:2164-77.

Wendling F, Benquet P, Bartolomei F, Jirsa V. Computational models of epileptiform activity. J Neurosci Methods. 2016;260:233-51.

Womelsdorf T, Valiante TA, Sahin NT, Miller KJ, Tiesinga P. Dynamic circuit motifs underlying rhythmic gain control, gating and integration. Nat Neurosci. 2014;17:1031-9.

Worrell GA, Gardner AB, Stead SM, Hu S, Goerss S, Cascino GJ, et al. High-frequency oscillations in human temporal lobe: simultaneous microwire and clinical macroelectrode recordings. Brain. 2008;131:928-37.

Wu SH, Ma CL, Kelly JB. Contribution of AMPA, NMDA, and GABA(A) receptors to temporal pattern of postsynaptic responses in the inferior colliculus of the rat. $\mathrm{J}$ Neurosci. 2004;24:4625-34.

Zelmann R, Zijlmans M, Jacobs J, Chatillon CE, Gotman J. Improving the identification of High Frequency Oscillations. Clin Neurophysiol. 2009;120:1457-64. 


\section{Figure Legends}

\section{Figure 1. The complete pipeline for HFO extraction from real depth-EEG recordings} performed during the presurgical evaluation of patients suffering from drug resistant epilepsy. (A) Sagittal view of the brain displaying the reconstructed entry points of intracerebral electrodes in one patient. The vertical plane containing electrode $C^{\prime}$ is also shown. (B) Coronal slice showing the intracerebral electrode C'. Electrodes are composed of 10-15 cylindrical contacts illustrated in red (length $2 \mathrm{~mm}$, diameter $0.8 \mathrm{~mm}$ and $1.5 \mathrm{~mm}$ apart) recording brain activity from deep to lateral structures. (C) Depth-EEG signals recorded on three intracerebral electrodes (A', B' and C') showing epileptic activities including HFOs. (D) Two typical examples of automatically-detected HFOs from 2 single channels. (E, F) Zoom on the two events shown in (D) to facilitate visual inspection.

Figure 2. Spatio-temporal model used to simulate epileptic HFOs. (A) Virtual brain environment in which the epileptic region is delineated inside a sulcus of the temporal lobe (green triangles). The surrounding tissue is considered to be a source of background activity (cyan triangles). (B) Each elementary triangle represents a neural population. Its temporal activity is modeled as a dipole (blue arrows) oriented perpendicularly to the triangle surface. The time-varying magnitude of each dipole is obtained from the physiological neuronal population model $\left(\mathrm{NP}_{\mathrm{k}}\right.$, where $\mathrm{k}$ denotes the $\mathrm{k}^{\text {th }}$ population) shown on the right. Each $\mathrm{NP}$ is composed of three types of subpopulations: (1) pyramidal cells (orange), (2) dendritic targeting interneurons (green) and (3) somatic targeting interneurons (yellow ellipse) which are further divided into interneurons mediating inhibitory GABAergic currents on pyramidal cells (blue), and interneurons mediating depolarizing GABAergic currents on pyramidal cells (red). (C) Intracerebral electrode inserted in the patch. The forward problem from NPs to 
electrode contacts is solved to simulate LFPs. (D) The afferent input to the cortical patch is modeled as set of signals $\mathrm{P}_{\mathrm{k}}(\mathrm{t})$ denoting incoming densities of action potentials (APs). In $\mathrm{P}_{\mathrm{k}} \mathrm{s}$, transient increases in the volleys of APs are represented as rectangular pulses that appear randomly in time according to a Gaussian distribution with mean $t_{0}$ (center of simulated HFO) and standard deviation $\sigma_{j}$ (where $j$ stands for "jitter"). $\sigma_{j}$ allows for controlling the synchronization level of the input. (E) Example of simulated HFO with corresponding slow and fast components.

Figure 3. Qualitative and quantitative comparison between real and simulated HFOs. (A) Three different types of real HFOs are compared to simulated ones. Each HFO is represented in both time-amplitude and time-frequency (spectrogram) domains. (B) Box plot representing the energy ratio between the high frequency component and the total energy for real and simulated HFOs. (C) Boxplot representing the normalized spectral entropy of real HFOs and simulated HFOs. (D) Boxplot of the modulation index characterizing the phaseamplitude coupling phenomenon for real and simulated HFOs. Plots in B, C and D were obtained from the same real $(n=100)$ and simulated $(n=360)$ HFOs.

Figure 4. Impact of model parameters on HFO features. (A) Effect of the amplitude of the dGABA PSP on HFO features: (1) the energy ratio of high frequency to total energy, (2) the median frequency, (3) the spectral entropy and (4) the standard deviation $(\sigma)$ of the bellshaped slow component computed on 1650 simulated HFOs. Each plot contains 15 different average values obtained from 110 HFOs simulated for each dGABA PSP amplitude value. (B) Effect of the percentage of neural populations (within the simulated patch) mediating dGABA PSPs onto PCs on the same four features (1-4). Each plot contains 15 different 
average values obtained from 110 HFOs simulated for each percentage value starting from $30 \%$ and increasing by $5 \%$. Note that below $30 \%$ no HFO is observed in simulated signals. (C) Effect of the jitter $\left(\sigma_{j}\right)$ on the same four features (1-4). Each plot contains 25 different average values obtained from 110 HFOs simulated for each value of jitter. The error bars in all plots represent the standard error (SEM). (D) Simulated signals as a function of the input jitter. Simulated and real signals for 3 jitter values are represented. For high jitter value (desynchronized input, $16 \mathrm{~ms}$ ), simulated signals resemble HFO events (left),for a medium jitter value $(9 \mathrm{~ms})$ the simulated signal exhibit half $\mathrm{HFO} /$ spike features and for low jitter value (synchronized inputs, $3 \mathrm{~ms}$ ) they become similar to real epileptic spikes (right).

Figure 5. Impact of the 3D source geometry on HFOs. (A) Simulated depth-EEG signals on 6 consecutive contacts of a virtual electrode inserted in a cortical gyrus. This case mimicks an open-field as the electric contribution of most neuronal populations, represented as blue dipoles, have approximately the same orientation, (B) Simulated depth-EEG signals on 6 consecutive contacts of a virtual electrode inserted between the banks of a sulcus. This case mimicks a closed field as the electric contribution of neuronal populations have opposite orientations, to a large extent. (C) Real signals on 6 consecutive contacts extracted from real depth-EEG recordings. Note that HFOs can be observed on several adjacent contacts and that HFOs can be clearly distinguished in the background activity, as predicted in the open field configuration. (D) Real signals extracted from depth-EEG recordings in another patient. Here HFOs appear only on 2 adjacent contacts. Fast ripples are less prominent w.r.t background, as predicted in the closed field configuration. (E) HFO to background ratio (HFO-to-BKG) as a function of space (along the electrode). "HFO-to-BKG" values were computed from 110 simulations of an open (red) and closed (blue) field case on 6 adjacent contacts. (F) "HFO-toBKG” values computed on real recordings on 6 adjacent contacts. 
Figure 6. The HFO model equation. (A) The simulation of realistic HFOs requires some conditions on time and space. Regarding time (B), HFOs stem from pathological depolarizing GABAergic synaptic potentials mediated by interneurons onto pyramidal cells in response to desynchronized volleys of action potentials (APs). Regarding space (C), open-field configurations favor the observability of HFOs w.r.t background activity. 


\section{ACCEPTED MANUSCRIPT}

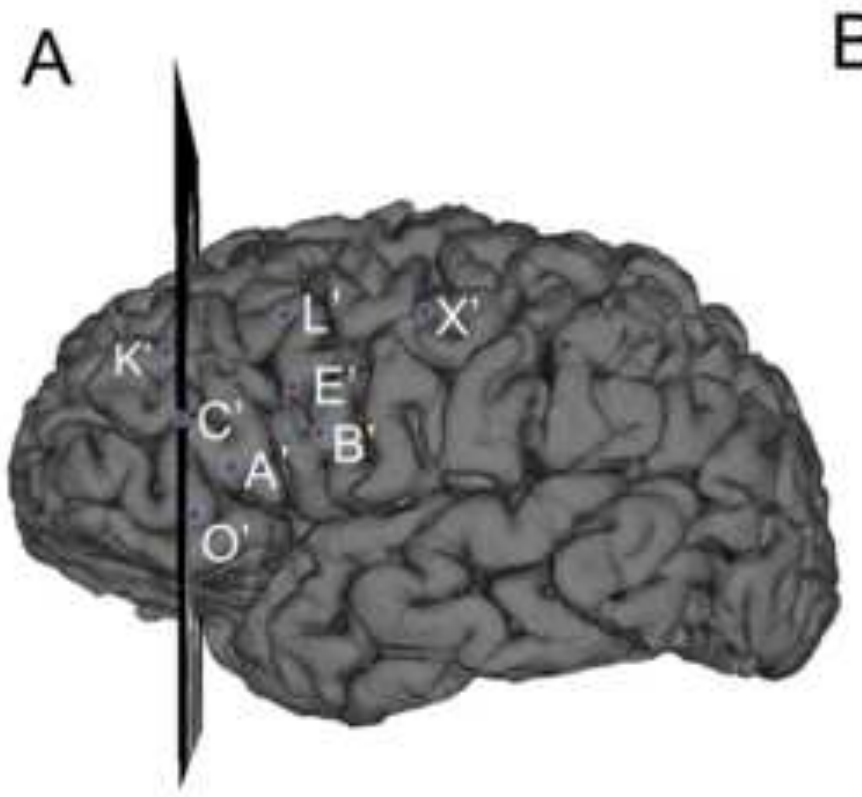

B

C
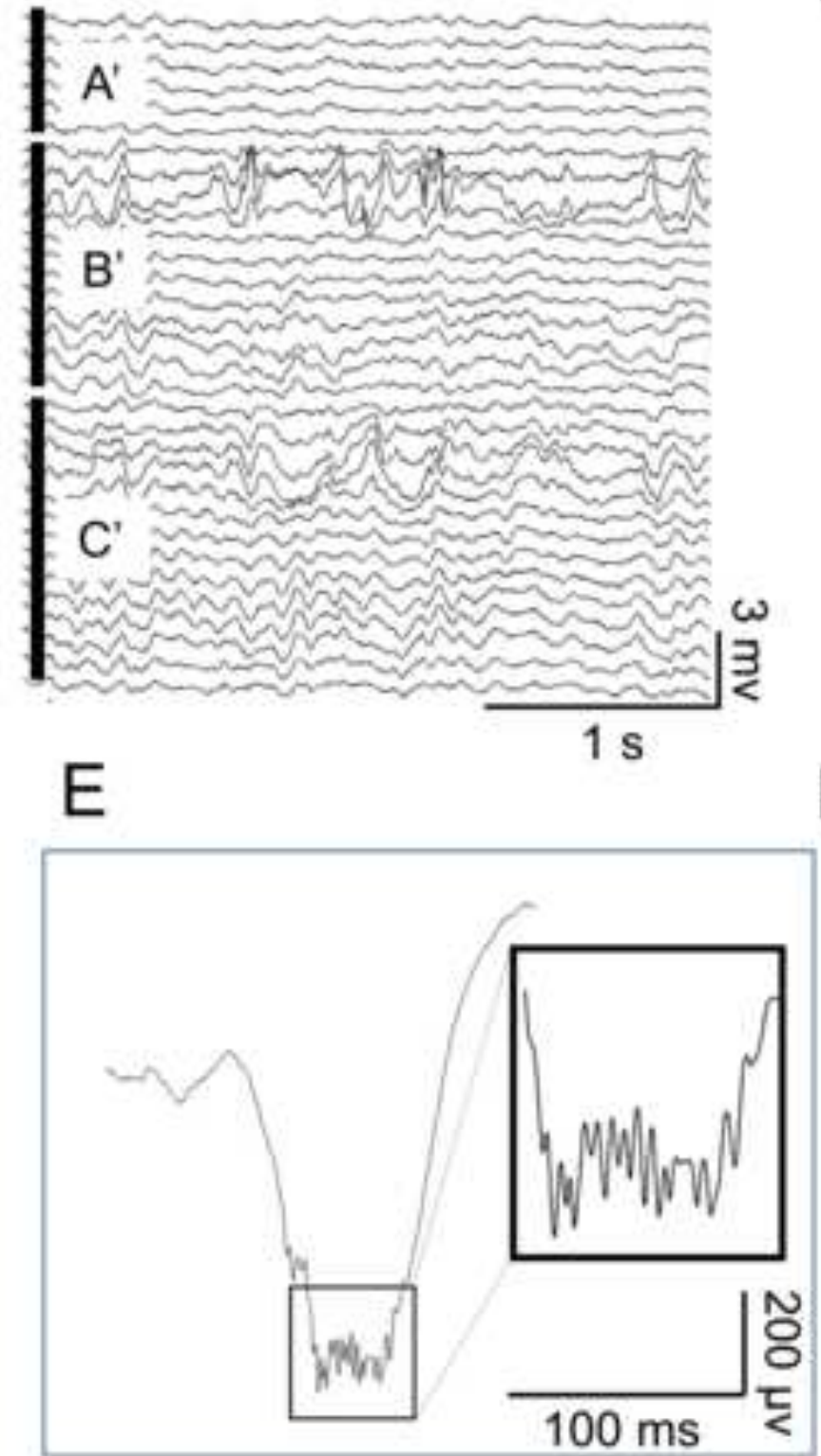

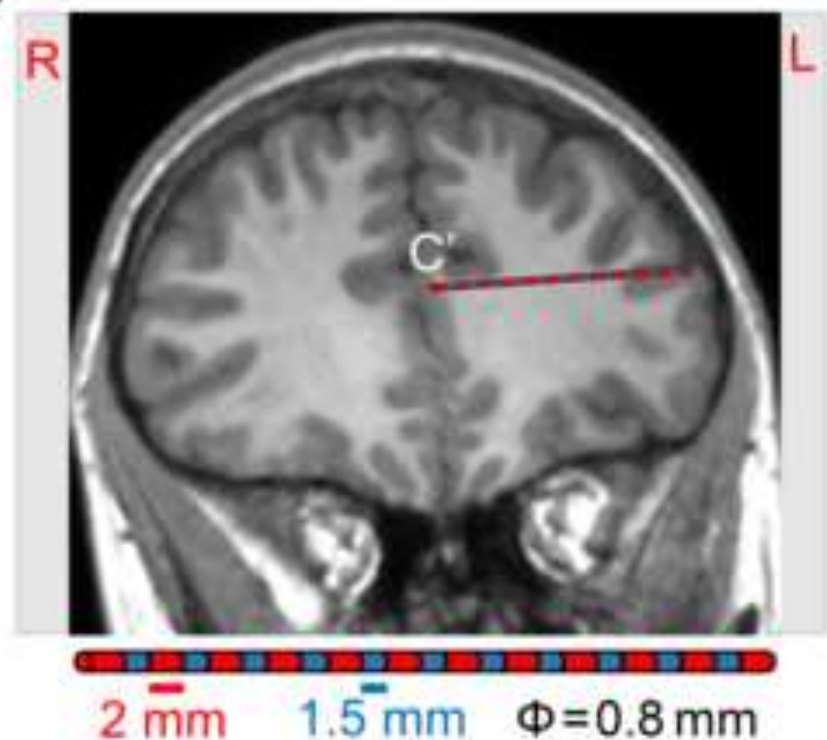

D

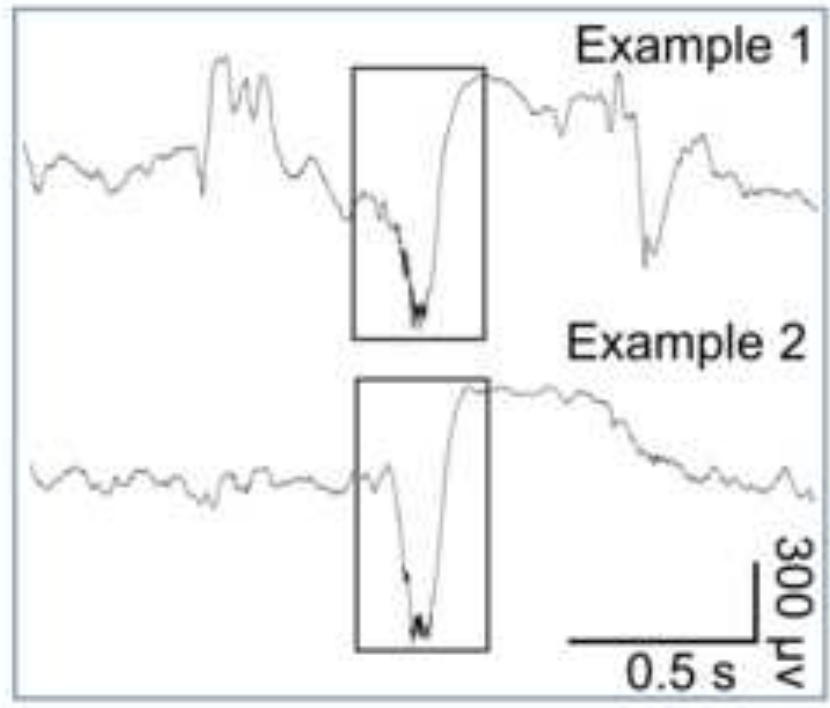

F

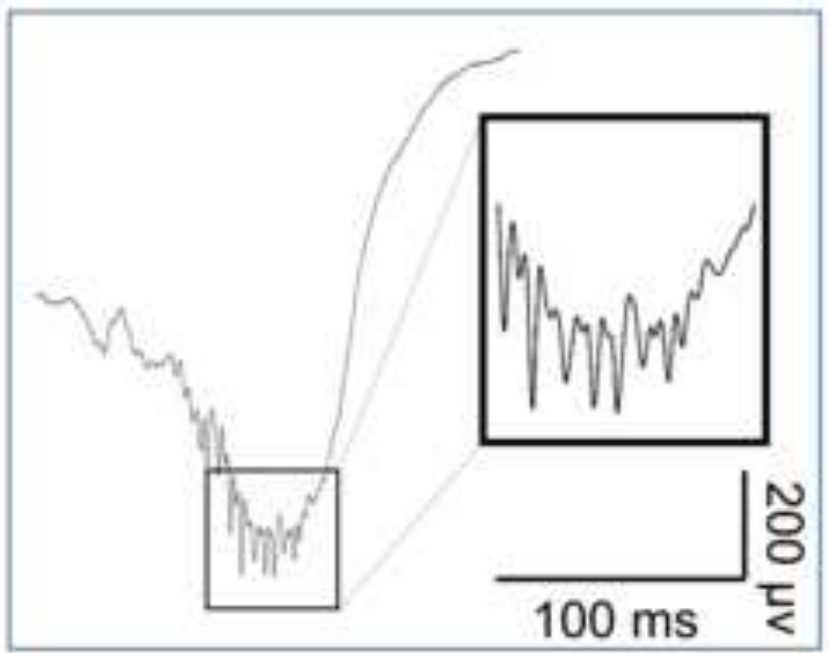




\section{ACCEPTED MANUSCRIPT}

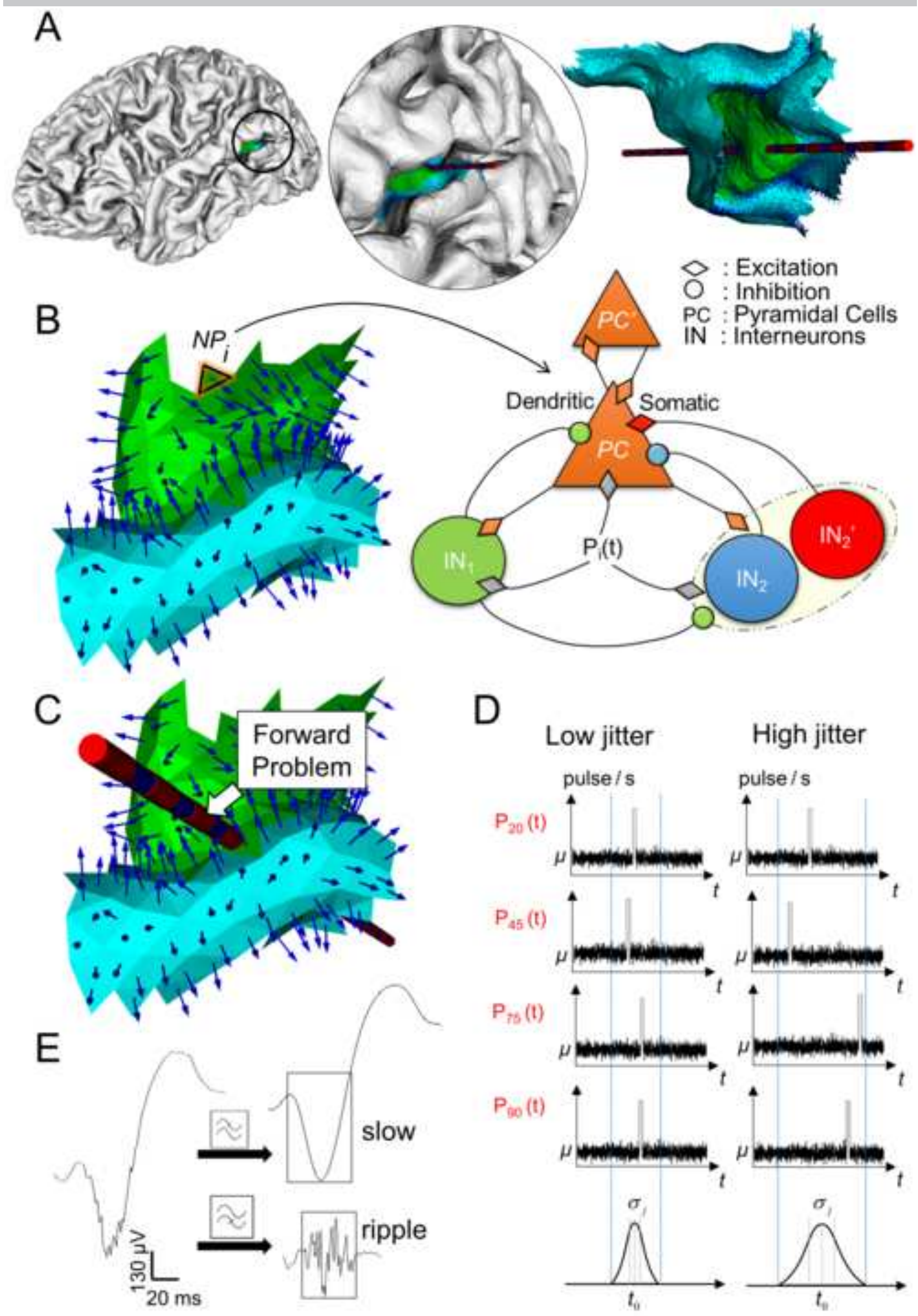


A Real HFOs Simulated HFOs B Real Simulated

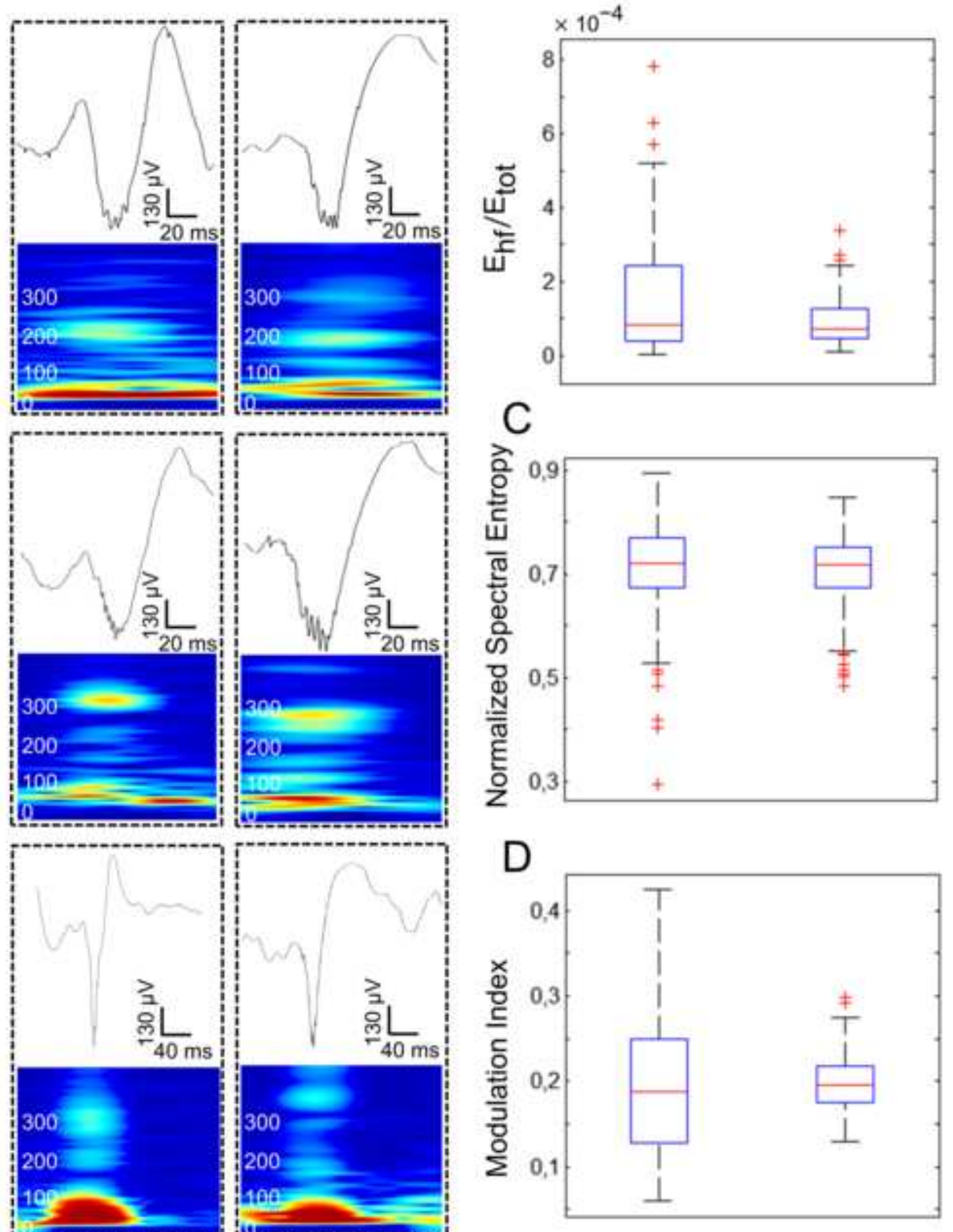




\section{ACCEPTED MANUSCRIPT}
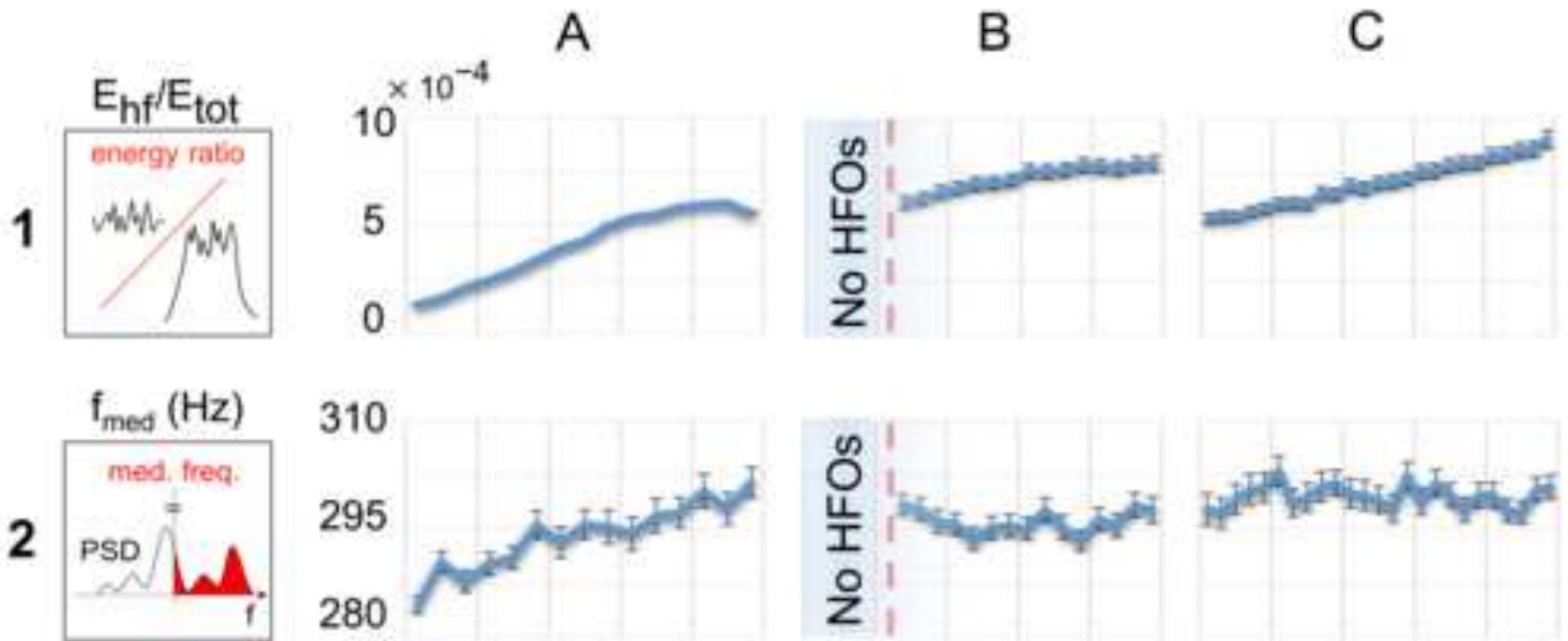

310
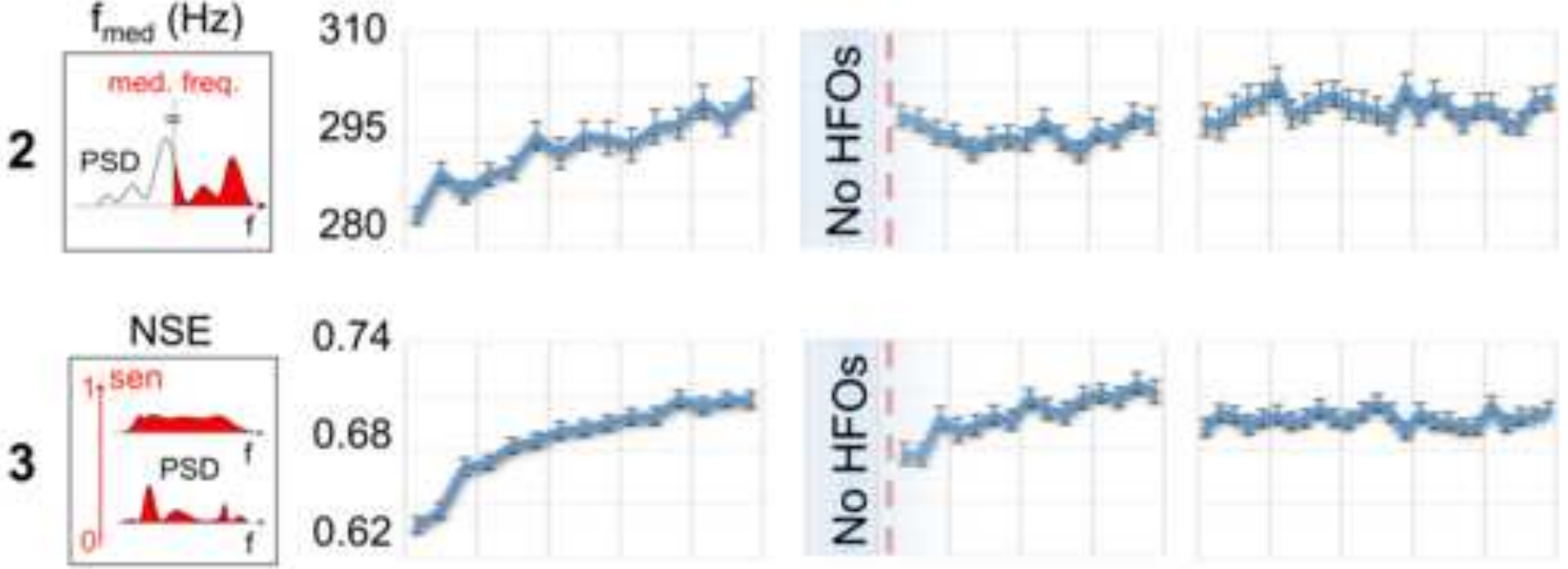

0.74

0.68
0.62
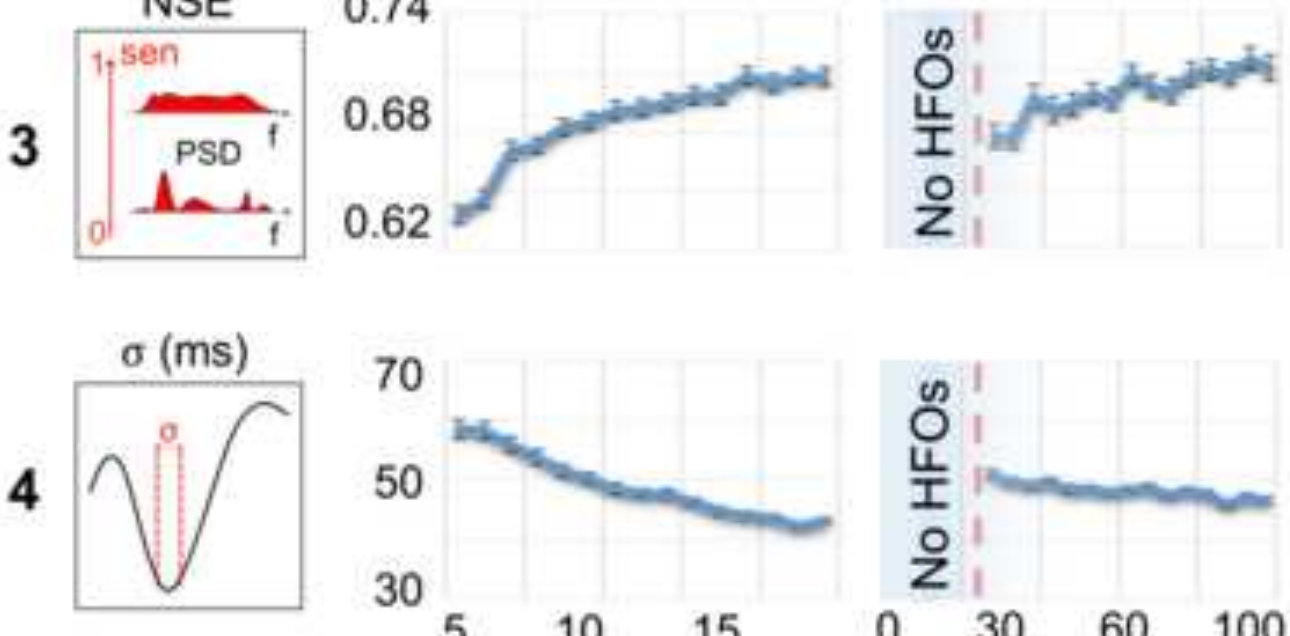

dGABA PSP Amplitude (mv)

$\%$ of NMs with dGABA
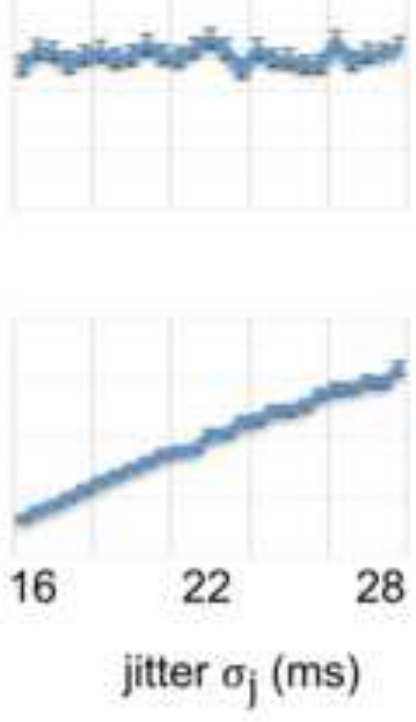

D

High Jitter HFOs ( $\sim 9 \mathrm{~ms})$

Spikes Low Jitter ( 16 ms)

$\frac{\frac{\mathrm{g}}{\frac{\pi}{3}}}{\frac{\mathrm{E}}{\omega}}$
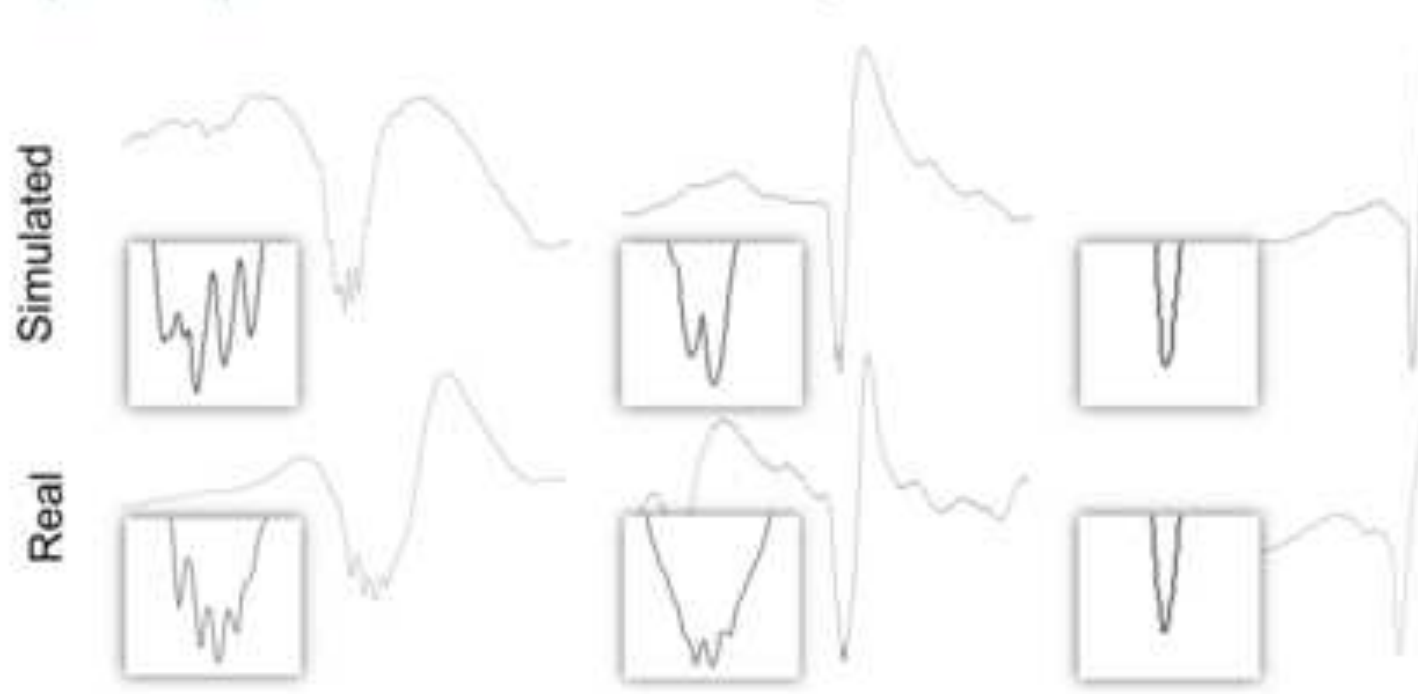


\section{ACCEPTED MANUSCRIPT}

A

Open field (simulated)

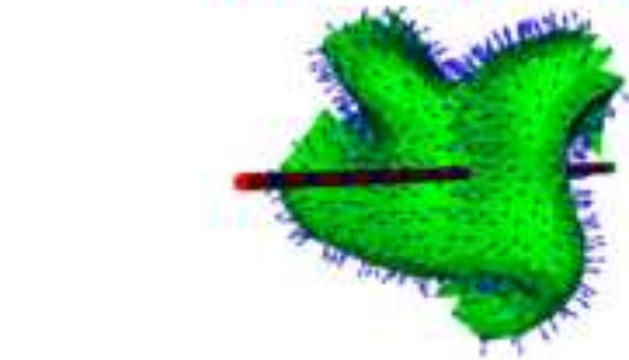

치이

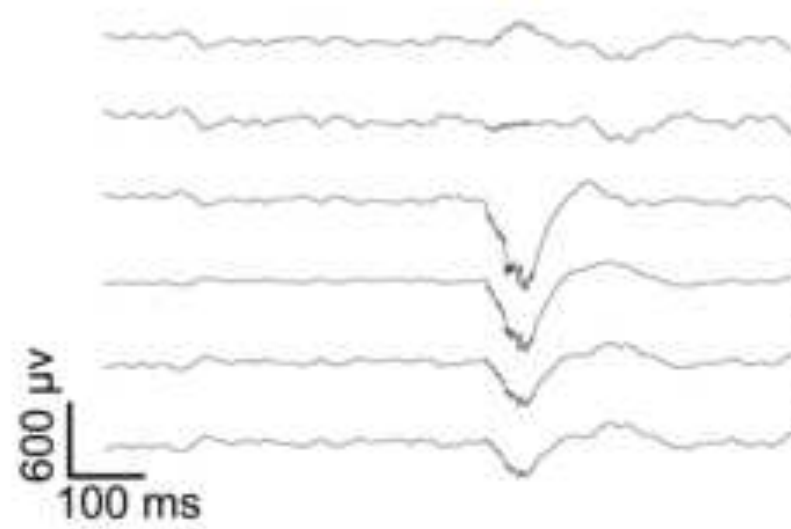

C

Open field (real)

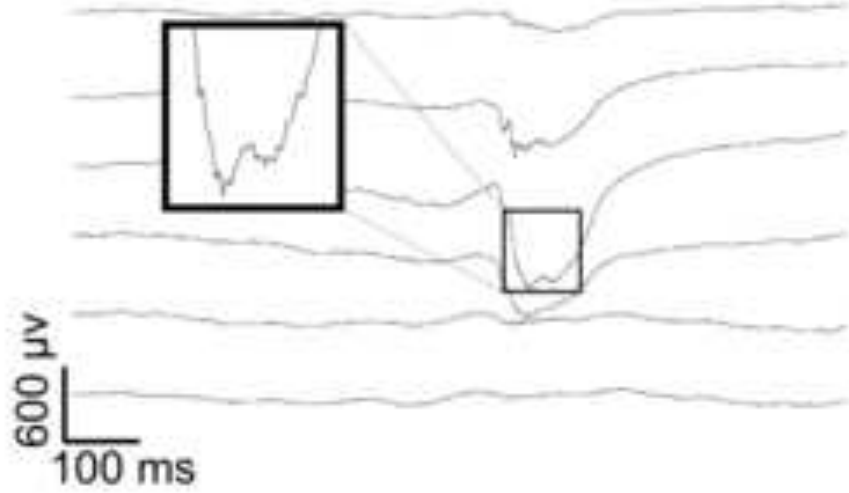

A

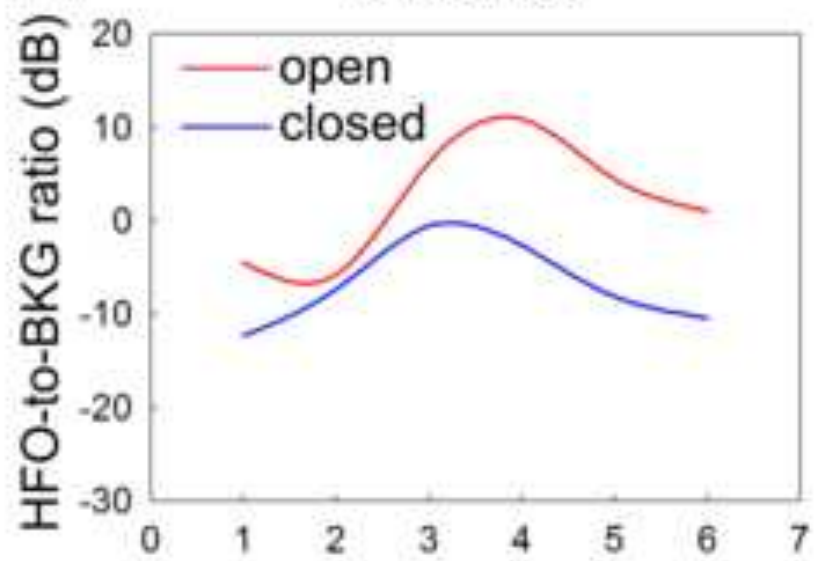

Electrode contact \#
\#0

Closed field (simulated)

$\# 1$
$\# 2$
$\# 3$
$\# 4$
$\# 5$
$\# 6$

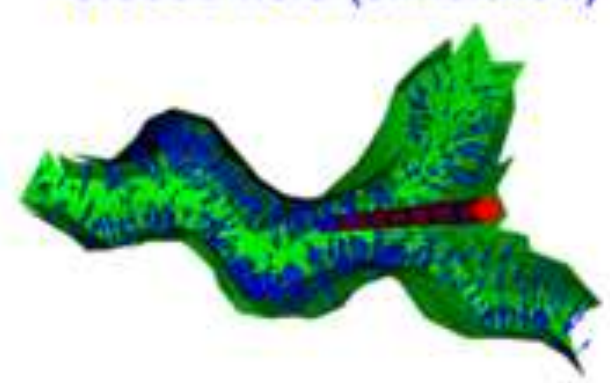

B
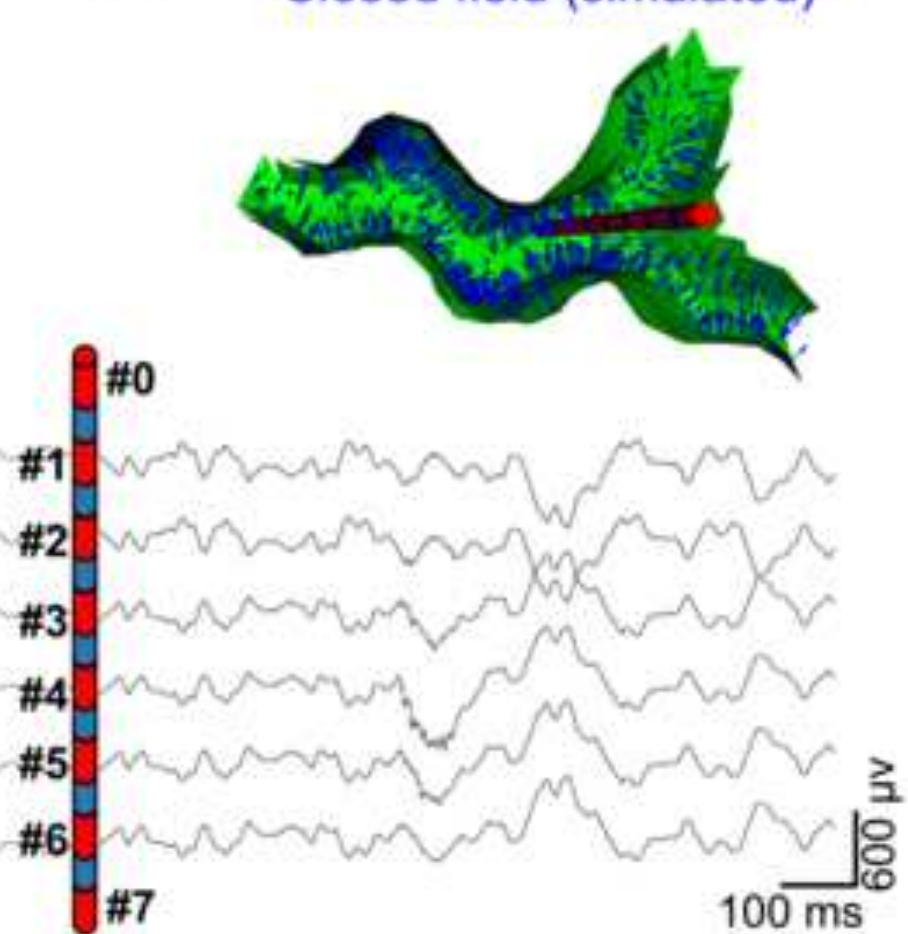

D Closed field (real)

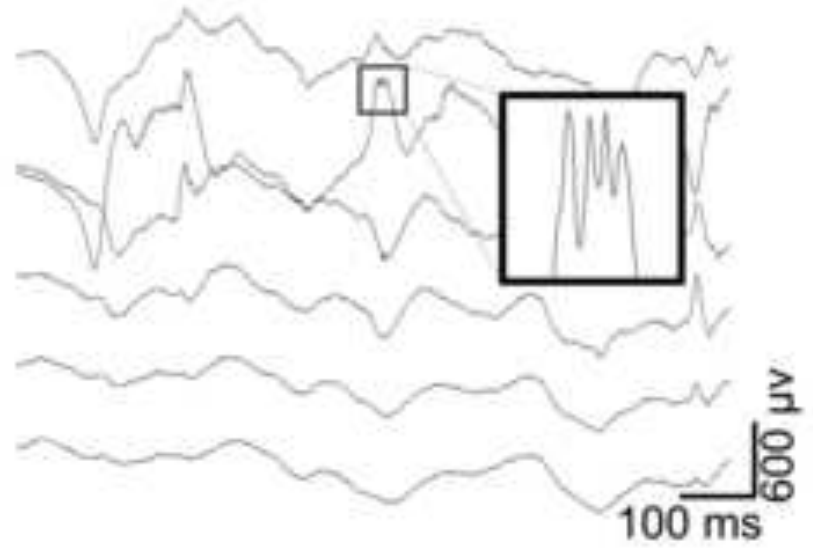

B

Real

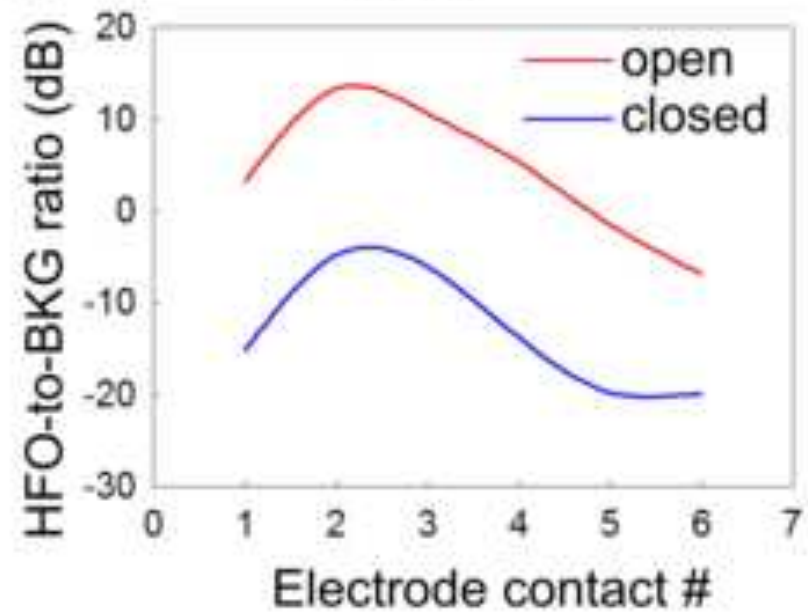




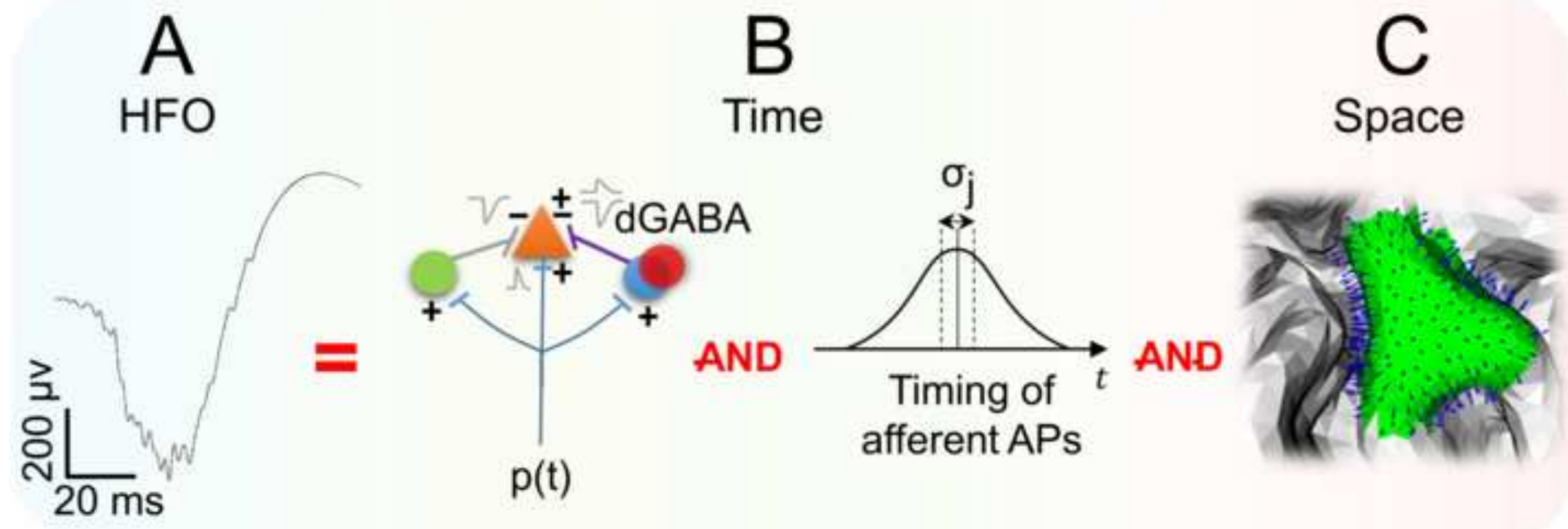


Table 1: model parameters interpretation and values.

\begin{tabular}{|c|c|c|c|}
\hline & Parameters & Significance & $\begin{array}{c}\text { Epileptic Tissue } \\
\text { (Background) }\end{array}$ \\
\hline 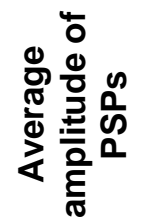 & $\begin{array}{l}\text { A (mv) } \\
B(m v) \\
G(m v) \\
J(m v)\end{array}$ & $\begin{array}{l}\text { Excitatory PSP of PCs } \\
\text { Inhibitory PSP of dendritic targeting INs } \\
\text { Inhibitory PSP of somatic targeting INs } \\
\text { Excitatory PSP of somatic targeting INs (dGABA) }\end{array}$ & $\begin{array}{c}3(1) \\
42(60) \\
5 \\
15(5)\end{array}$ \\
\hline 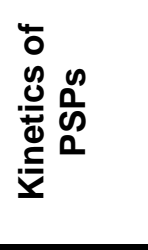 & $\begin{array}{l}a\left(s^{-1}\right) \\
b\left(s^{-1}\right) \\
g_{r}\left(s^{-1}\right) \\
g_{d}\left(s^{-1}\right)\end{array}$ & $\begin{array}{l}\text { Rate constant of PSP of PCs } \\
\text { Rate constant of PSP of dendritic targeting INs } \\
\text { Rate constant for rise time of PSP of somatic targeting INs } \\
\text { Rate constant for decay time of PSP of somatic targeting INs }\end{array}$ & $\begin{array}{c}100 \\
33 \\
3000 \\
400\end{array}$ \\
\hline 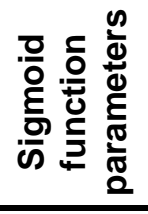 & $\begin{array}{l}e_{0}(\text { pulse/s) } \\
v_{0}(m v) \\
r\left(m v^{-1}\right)\end{array}$ & $\begin{array}{l}\text { Maximum firing rate (saturation value) } \\
\text { Threshold average membrane potential } \\
\text { Steepness of the linear part of sigmoid function }\end{array}$ & $\begin{array}{c}2.5 \\
6 \\
0.56\end{array}$ \\
\hline 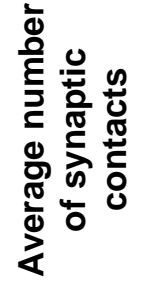 & $\begin{array}{l}C_{P, P}, C_{P, P} \\
C_{P, N_{1}}, C_{\mathbb{I N}_{1}, P} \\
C_{P, \mathbb{N}_{2}}, C_{\mathbb{I N}_{2}, P} \\
C_{\mathbb{I N}_{1}, N_{2}}\end{array}$ & $\begin{array}{l}\text { Between PCs } \\
\text { Between PCs and dendritic targeting INs } \\
\text { Between PCs and somatic targeting INs } \\
\text { Between somatic targeting and dendritic targeting INs }\end{array}$ & $\begin{array}{c}135,110 \\
27,33 \\
135,135 \\
15\end{array}$ \\
\hline 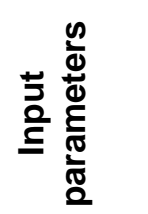 & $\begin{array}{l}\Delta \overline{\mathrm{AP}} \\
\sigma_{\mathrm{j}}(\mathrm{ms}) \\
\sigma_{\mathrm{n}}(\text { pulse } / \mathrm{s}) \\
\mu \text { (pulse/s) }\end{array}$ & $\begin{array}{l}\text { Samples of variation in afferent density of action potentials } \\
\text { Standard deviation of jitter } \\
\text { Standard deviation of input density of action potentials } \\
\text { mean of input density of action potentials }\end{array}$ & $\begin{array}{c}5(0) \\
25(0) \\
0.1 \\
3\end{array}$ \\
\hline 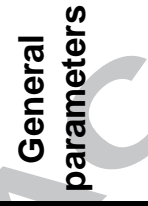 & $\begin{array}{l}T(s) \\
f_{s}(H z) \\
f_{d}(H z)\end{array}$ & $\begin{array}{l}\text { Duration of simulation } \\
\text { Sampling frequency } \\
\text { Down-sampling frequency }\end{array}$ & $\begin{array}{c}2 \\
8192 \\
2048\end{array}$ \\
\hline
\end{tabular}

